\title{
Bochner integrals in ordered vector spaces
}

\author{
A. C. M. van Rooij ${ }^{1}$ • W. B. van Zuijlen ${ }^{2}$
}

Received: 21 April 2016 / Accepted: 31 October 2016 / Published online: 1 December 2016 C The Author(s) 2016. This article is published with open access at Springerlink.com

\begin{abstract}
We present a natural way to cover an Archimedean directed ordered vector space $E$ by Banach spaces and extend the notion of Bochner integrability to functions with values in $E$. The resulting set of integrable functions is an Archimedean directed ordered vector space and the integral is an order preserving map.
\end{abstract}

Keywords Bochner integral · Ordered vector space $\cdot$ Ordered Banach space $\cdot$ Closed cone $\cdot$ Generating cone

Mathematics Subject Classification 28B05 $\cdot$ 28B15

\section{Introduction}

We extend the notion of Bochner integrability to functions with values in a vector space $E$ that may not itself be a Banach space but is the union of a collection $\mathbb{B}$ of Banach spaces.

The idea is the following. We call a function $f$, defined on a measure space $X$ and with values in $E$, "integrable" if for some $D$ in $\mathbb{B}$ all values of $f$ lie in $D$ and $f$ is Bochner integrable as a function $X \rightarrow D$. Of course, one wants a certain consistency: the "integral" of such an $f$ should be independent of the choice of $D$.

In [18], Thomas obtains this consistency by assuming a Hausdorff locally convex topology on $E$, entailing many continuous linear functions $E \rightarrow \mathbb{R}$. Their restrictions

\footnotetext{
$\varangle \quad$ W. B. van Zuijlen vanzuijlen@wias-berlin.de

1 Department of Mathematics, Radboud University Nijmegen, P.O. Box 9010, 6500 GL Nijmegen, The Netherlands

2 Mathematical Institute, Leiden University, P.O. Box 9512, 2300 RA Leiden, The Netherlands
} 
to the Banach spaces that constitute $\mathbb{B}$ enable one to apply Pettis integration, which leads to the desired uniqueness.

Our approach is different, following a direct-limit-like construction. We assume $E$ to be an ordered vector space with some simple regularity properties (Archimedean, directed) and show that $E$ is the union of a certain increasing system $\mathbb{B}$ of Banach spaces with closed, generating positive cones (under the ordering of $E$ ). Uniqueness of the integral follows from properties of such ordered Banach spaces. Moreover, the integrable functions form a vector space and the integral is linear and order preserving.

In Sect. 3 we study ordered Banach spaces with closed generating cones. We give certain properties which can be used to give an alternative proof of a classical theorem which states that every order preserving linear map is continuous, and generalise it to order bounded linear maps. In Sect. 4 we study Bochner integrable functions with values in an ordered Banach space with closed generating cone. In Sect. 5 we present the definition of a Banach cover and the definition of the extension of the Bochner integral to functions with values in a vector space that admits a Banach cover. In Sect. 6 we show that an Archimedean ordered vector space possesses a Banach cover consisting of ordered Banach spaces with closed generating cones. In Sect. 7 we study integrable functions with values in Archimedean ordered vector spaces. In Sect. 8 we compare the integral with integrals considered in [16]. In Sect. 9 we present an application to view the convolution as an integral.

\section{Notation}

$\mathbb{N}$ is $\{1,2, \ldots\}$. We write "for all $n$ " instead of "for all $n \in \mathbb{N}$ ". To avoid confusion:

- An "order" is a "partial order".

- We call an ordered vector space Archimedean (see Peressini [15]) if for all $a, b \in E$ the following holds: if $n a \leq b$ for all $n \in \mathbb{N}$, then $a \leq 0$. (In some places, e.g., Birkhoff [4], such spaces are said to be 'integrally closed'.)

As is common in literature, our notations do not distinguish between a function on a measure space and the class of that function.

\section{Ordered Banach spaces with closed generating cones}

In this section we describe properties of ordered Banach spaces with closed generating cones. Using these properties we prove in Theorem 3.11 that an order bounded map between ordered Banach spaces with closed generating cones is continuous.

Definition 3.1 An ordered locally convex vector space is a locally convex vector space with an order that makes it an ordered vector space. A normed ordered vector space is a normed vector space with an order that makes it an ordered vector space. An ordered Banach space is a Banach space that is a normed ordered vector space.

A priori there is no connection between the ordering and the topology of an ordered locally convex vector space. One reasonable and useful connection is the assumption that the (positive) cone be closed. 
Theorem 3.2 Let $E$ be an ordered locally convex vector space. $E^{+}$is closed if and only if

$$
x \leq y \Longleftrightarrow \alpha(x) \leq \alpha(y) \text { for all } \alpha \in\left(E^{\prime}\right)^{+} .
$$

Consequently, whenever $E^{+}$is closed then $\left(E^{\prime}\right)^{+}$separates the points of $E$ and $E$ is Archimedean.

Proof Since $E^{+}$is convex, $E^{+}$is closed if and only if it is weakly closed (i.e., $\sigma\left(E, E^{\prime}\right)$-closed); see [7, Theorem V.1.4]. The rest follows by [1, Theorem 2.13 (3\& $4)]$.

The following theorem is due to Andô [2]. See also [1, Corollary 2.12].

Theorem 3.3 Let D be an ordered Banach space with a closed generating ${ }^{1}$ cone $D^{+}$. There exists a $C>0$ such that

$$
C\|x\| \geq \inf \left\{\|a\|: a \in D^{+},-a \leq x \leq a\right\} \quad(x \in E) .
$$

Definition 3.4 Let $D$ be a directed ${ }^{2}$ ordered Banach space. If $C>0$ is such that (2) holds, then we say that the norm $\|\cdot\|$ is $C$-absolutely dominating. ${ }^{3}$ We say that a norm $\|\cdot\|$ is absolutely dominating if it is $C$-absolutely dominating for some $C>0$.

On a Banach lattice the norm is 1-absolutely dominating. Actually for Banach lattices there is equality in (2).

3.5 We refer the reader to Appendix 1 for the following facts: If $\|\cdot\|$ is $C$-absolutely dominating on a directed ordered Banach space $D$, then $C \geq 1$. Whenever there exists a absolutely dominating norm, then for all $\varepsilon>0$ there exists an equivalent $(1+\varepsilon)$ absolutely dominating norm. All norms on a directed ordered vector space $D$ that make $D$ complete and $D^{+}$closed are equivalent (see 6.2).

3.6 Let $D$ be a directed ordered Banach space. Then $\|\cdot\|$ is absolutely dominating if and only if the (convex) set

$$
\bigcup_{a \in D^{+},\|a\| \leq 1}[-a, a]
$$

is a neighbourhood of 0 .

Definition 3.7 (See $[12, \S 16]$ ) Let $E$ be an ordered vector space. We say that a sequence $\left(x_{n}\right)_{n \in \mathbb{N}}$ in $E$ converges uniformly to an element $x \in E$ (notation: $x_{n} \stackrel{u}{\rightarrow} x$ ) whenever there exist $a \in E^{+}, \varepsilon_{n} \in(0, \infty)$ with $\varepsilon_{n} \rightarrow 0$ and

$$
-\varepsilon_{n} a \leq x_{n}-x \leq \varepsilon_{n} a \quad(n \in \mathbb{N}) .
$$

\footnotetext{
$1 D^{+}$is generating if $D=D^{+}-D^{+}$.

$2 D$ is directed if $D=D^{+}-D^{+}$, i.e., if $D^{+}$is generating.

3 Batty and Robinson [3] call the cone $D^{+}$approximately $C$-absolutely dominating, and, Messerschmidt [13] calls $D$ approximately $C$-absolutely conormal, if the norm on $D$ is $C$-absolutely dominating.
} 
Note that one may replace " $\varepsilon_{n} \rightarrow 0$ " by " $\varepsilon_{n} \downarrow 0$ ". If $E$ is Archimedean then the $x$ as above is unique. We will only consider such convergence in Archimedean spaces. We say that a sequence $\left(x_{n}\right)_{n \in \mathbb{N}}$ in $E$ is a uniformly Cauchy sequence if there exists an $a \in E^{+}$such that for all $\varepsilon>0$ there exists an $N$ such that $-\varepsilon a \leq x_{n}-x_{m} \leq \varepsilon a$ for all $n, m \geq N$. E is called uniformly complete whenever it is Archimedean and all uniformly Cauchy sequences converge uniformly.

Lemma 3.8 Let $D_{1}, D_{2}$ be ordered vector spaces and $T: D_{1} \rightarrow D_{2}$. If $T$ is linear and order bounded, then $T$ preserves uniform convergence.

Proof Suppose $x_{n} \in D_{1}, \varepsilon_{n} \in(0,1)$ and $a \in D_{1}^{+}$are such that $-\varepsilon_{n} a \leq x_{n} \leq \varepsilon_{n} a$ and $\varepsilon_{n} \downarrow 0$. Let $b \in D_{2}^{+}$be such that $T[-a, a] \subset[-b, b]$. Then $\varepsilon_{n}^{-1} T x_{n} \in[-b, b]$, i.e., $-\varepsilon_{n} b \leq T x_{n} \leq \varepsilon_{n} b$ for all $n$.

Theorem 3.9 Let D be an ordered Banach space. Consider the following conditions.

(i) $\|\cdot\|$ is absolutely dominating.

(ii) If $a_{1}, a_{2}, \ldots \in D^{+}, \sum_{n \in \mathbb{N}}\left\|a_{n}\right\|<\infty$, then there exist $a \in D^{+}, \varepsilon_{n} \in(0, \infty)$ with $\varepsilon_{n} \rightarrow 0$ such that $a_{n} \leq \varepsilon_{n}$ a for all $n .^{4}$

(iii) If $x_{1}, x_{2}, \ldots \in D, \sum_{n \in \mathbb{N}}\left\|x_{n}\right\|<\infty$, then $x_{n} \stackrel{u}{\rightarrow} 0$.

If $D^{+}$is closed, then $D$ satisfies (ii).

Suppose D is Archimedean and directed. Then the following are equivalent.

(a) $D^{+}$is closed.

(b) D satisfies (i) and (ii).

(c) D satisfies (iii)

Proof Suppose $D^{+}$is closed. Let $a_{1}, a_{2}, \ldots \in D^{+}, \sum_{n \in \mathbb{N}}\left\|a_{n}\right\|<\infty$. Choose $\varepsilon_{n} \in(0, \infty), \varepsilon_{n} \rightarrow 0$ such that $\sum_{n \in \mathbb{N}} \varepsilon_{n}^{-1}\left\|a_{n}\right\|<\infty$. By norm completeness, $a:=\sum_{n \in \mathbb{N}} \varepsilon_{n}^{-1} a_{n}$ exists. Because $D^{+}$is closed $a \geq \varepsilon_{n}^{-1} a_{n}$ for all $n$.

(a) $\Longrightarrow$ (b). (ii) is implied by the above argument. By Theorem 3.3 we have (i).

(b) $\Longrightarrow$ (c). Let $x_{1}, x_{2}, \ldots \in D, \sum_{n \in \mathbb{N}}\left\|x_{n}\right\|<\infty$. Using (i) let $a_{1}, a_{2}, \ldots \in D^{+}$ with $\sum_{n \in \mathbb{N}}\left\|a_{n}\right\|<\infty$ be such that $-a_{n} \leq x_{n} \leq a_{n}$. By (ii) it then follows that $x_{n} \stackrel{u}{\rightarrow} 0$.

(c) $\Longrightarrow$ (a). Take $b$ in the closure of $D^{+}$. For $n \in \mathbb{N}$, choose $x_{n} \in D^{+}, \sum_{n \in \mathbb{N}} \| x_{n}-$ $b \|<\infty$. By (iii) there exist $a \in D^{+}, \varepsilon_{n} \in(0, \infty)$ with $\varepsilon_{n} \rightarrow 0$ and $-\varepsilon_{n} a \leq x_{n}-b \leq$ $\varepsilon_{n} a$, so that $b \geq x_{n}-\varepsilon_{n} a \geq-\varepsilon_{n} a$. Then $b \geq 0$ because $D$ is Archimedean.

Lemma 3.10 Let $D$ be an ordered Banach space for which $D^{+}$is closed. Then

$$
x_{1}, x_{2}, \ldots \in D, a \in D, b \in D,\left\|x_{n}-a\right\| \rightarrow 0, x_{n} \stackrel{u}{\rightarrow} b \Longrightarrow a=b .
$$

Proof Assume $b=0$. There exist $c \in D^{+}, \varepsilon_{n} \in(0, \infty)$ with $\varepsilon_{n} \downarrow 0$ and $-\varepsilon_{n} c \leq x_{n} \leq$ $\varepsilon_{n} c$. Then $-\varepsilon_{N} c \leq x_{n} \leq \varepsilon_{N} c$ whenever $n \geq N$. Since $D^{+}$is closed, $-\varepsilon_{N} c \leq a \leq \varepsilon_{N} c$ for all $N$, and so, as $D$ is Archimedean by Theorem 3.2, $a=0$.

\footnotetext{
${ }^{4}$ For normed Riesz spaces property (ii) is equivalent to what is called the weak Riesz-Fischer condition (see Zaanen [19, Ch. 14, §101]).
} 
With this we can easily prove the following theorem. In a recently published book by Aliprantis and Tourky [1] ${ }^{5}$ but also in older Russian papers by Wulich [5] one can find the proof that an order preserving linear map is continuous (see [1, Theorem 2.32] or combine [5, Theorem III.2.2] (which states the result for $D_{2}=\mathbb{R}$ ) and [6, Theorem VI.2.1]). Theorem 3.11 is more general in the sense that it states that linear order bounded maps are continuous.

Theorem 3.11 Let $D_{1}, D_{2}$ be ordered Banach spaces. Suppose $D_{1}^{+}$is closed and generating and $D_{2}^{+}$is closed. Let $T: D_{1} \rightarrow D_{2}$ be linear and order bounded. Then $T$ is continuous. Consequently, if $T$ is an order isomorphism then it is a homeomorphism.

Proof Let $x_{1}, x_{2}, \cdots \in D_{1}, x_{n} \rightarrow 0$ and suppose $T x_{n} \rightarrow c$ for some $c \in D_{2}$. If from this we can prove $c=0$, then by the Closed Graph Theorem $T$ will be continuous. We may assume $\sum_{n \in \mathbb{N}}\left\|x_{n}\right\|<\infty$. Then $x_{n} \stackrel{u}{\rightarrow} 0$ in $D_{1}$ (Theorem 3.9), so $T x_{n} \stackrel{u}{\rightarrow} 0$ in $D_{2}$ (Lemma 3.8). Hence, $c=0$ according to Lemma 3.10.

We present a consequence as has been done for order preserving linear maps in [6, Theorem VI.2.2].

Corollary 3.12 Let $D_{1}$ be an ordered Banach space with closed generating cone. Let $D_{2}$ be an ordered normed vector space with a normal cone. Let $T: D_{1} \rightarrow D_{2}$ be linear and order bounded. Then $T$ is continuous.

Proof As $D_{2}^{+}$is normal, we have $D_{2}^{\sim}=D_{2}^{\prime}$ (see [1, Corollary 2.27]). Let $\Delta$ be the unit ball in $D_{1}$. Then for all $\phi \in D_{2}^{\prime}$, the map $\phi \circ T$ is an order bounded functional, whence continuous by Theorem 3.11. Thus $\phi \circ T(\Delta)$ is bounded for all $\phi \in D_{2}^{\prime}$. By an application of the Principle of Uniform Boundedness (see [7, Corollary III.4.3]), $T(\Delta)$ is norm bounded.

Theorem 3.13 [14, Theorem 6.8] Let D be an ordered Banach space with closed cone $D^{+}$. If $f \in D^{\prime}$ and $f \in D^{\sim}$, i.e., $f$ is linear continuous and order bounded, then $f$ is regular, i.e., there are $f_{1}, f_{2} \in D^{\sim+}$ with $f=f_{1}-f_{2}$.

By Theorem 3.13 and Theorem 3.11 we derive the following corollary.

Corollary 3.14 Let D be an ordered Banach space with closed generating cone. Then every order bounded linear map $D \rightarrow \mathbb{R}$ is regular, i.e., $D^{\sim}=D^{\sim+}-D^{\sim+}$.

\section{The Bochner integral on ordered Banach spaces}

\section{In this section $(X, \mathcal{A}, \mu)$ is a complete $\sigma$-finite measure space, with $\mu \neq 0$. For more assumptions see 4.3 and 4.7 .}

We define the integral of simple functions in Definition 4.1 and recall the definition and facts on Bochner integrability in Definitions 4.2 and 4.4. After that, we consider an ordered Banach space $D$. In Theorems 4.5 and 4.6 we describe the order structure of the space $\mathfrak{B}_{D}$ of Bochner integrable functions. In 4.8 we summarise the results of 4.9-4.16, in which we compare closedness and generatingness of the positive cones of $D$ and $\mathfrak{B}_{D}$.

\footnotetext{
${ }^{5}$ For complete metrisable ordered vector spaces.
} 
Definition 4.1 Let $E$ be a vector space. We say that a function $f: X \rightarrow E$ is simple if there exist $N \in \mathbb{N}, a_{1}, \ldots, a_{N} \in E, A_{1}, \ldots, A_{N} \in \mathcal{A}$ with $\mu\left(A_{1}\right), \ldots, \mu\left(A_{N}\right)<\infty$ for which

$$
f=\sum_{n=1}^{N} a_{n} \mathbb{1}_{A_{n}} .
$$

The simple functions form a linear subspace $S$ of $E^{X}$, which is a Riesz subspace of $E^{X}$ in case $E$ is a Riesz space. We define $\varphi: S \rightarrow E$ by

$$
\varphi(f)=\sum_{n=1}^{N} \mu\left(A_{n}\right) a_{n}
$$

where $f, N, A_{n}, a_{n}$ are as in (6). $\varphi(f)$ is called the integral of $f$. We write $S_{\mathbb{R}}$ for the linear space of simple functions $X \rightarrow \mathbb{R}$.

Definition 4.2 and the facts in 4.4 can be found in Chapter III in the book by Hille and Phillips [9].

Definition 4.2 Let $(D,\|\cdot\|)$ be a Banach space. A function $f: X \rightarrow D$ is called Bochner integrable whenever there exists a sequence of simple functions $\left(s_{n}\right)_{n \in \mathbb{N}}$ such that $s_{n}(x) \rightarrow f(x)$ for $\mu$-almost all $x \in X$ and

$$
\int\left\|f(x)-s_{n}(x)\right\| \mathrm{d} \mu(x) \rightarrow 0 .
$$

Then the sequence $\left(\varphi\left(s_{n}\right)\right)_{n \in \mathbb{N}}$ converges. Its limit is independent of the choice of the sequence $\left(s_{n}\right)_{n \in \mathbb{N}}$ and is called the Bochner integral of $f$.

4.3 For the rest of this section, $D$ is a Banach space (with norm $\|\cdot\|$ ), and, we write $\mathfrak{B}$ (or) $\mathfrak{B}_{D}$ for the Banach space of classes of Bochner integrable functions $X \rightarrow D$, with norm $\|\cdot\|_{\mathfrak{B}}$ (see 4.4(b)). We write $\mathfrak{b}($ or $) \mathfrak{b}_{D}$ for the Bochner integral on $\mathfrak{B}$.

4.4 Some facts on the Bochner integrable functions:

(a) [9, Theorem 3.7.4] and [17, Proposition 2.15] If $f: X \rightarrow D$ is Borel measurable, $f(X)$ is separable and $\int\|f\| \mathrm{d} \mu<\infty$, then $f$ is Bochner integrable.

(b) $\left[9\right.$, Theorem 3.7.8] $\mathfrak{B}$ is a Banach space under the norm $\|\cdot\|_{\mathfrak{B}}: \mathfrak{B} \rightarrow[0, \infty)$ given by

$$
\|f\|_{\mathfrak{B}}=\int\|f\| \mathrm{d} \mu
$$

(c) [9, Theorem 3.7.12] Let $E$ be a Banach space and $T: D \rightarrow E$ be linear and continuous. If $f \in \mathfrak{B}_{D}$, then $T \circ f \in \mathfrak{B}_{E}$ and

$$
T\left(\mathfrak{b}_{D}(f)\right)=\mathfrak{b}_{E}(T \circ f) .
$$


(d) [9, Theorem 3.7.9] Let $f_{1}, f_{2}, \ldots$ be in $\mathfrak{B}, f: X \rightarrow D$ and $h \in \mathcal{L}^{1}(\mu)^{+}$. Suppose that $f_{n}(x) \rightarrow f(x)$ and $\left\|f_{n}(x)\right\| \leq h(x)$ for $\mu$-almost all $x \in X$. Then $f \in \mathfrak{B}$ and

$$
\mathfrak{b}\left(f_{n}\right) \rightarrow \mathfrak{b}(f)
$$

(e) If $D$ is an ordered Banach space then $\mathfrak{B}$ is an ordered Banach space under the ordering given by

$$
f \leq g \quad(\text { in } \mathfrak{B}) \Longleftrightarrow f \leq g \quad \mu-\text { a.e.. }
$$

Theorem 4.5 Let $D$ be a Banach lattice. Then $\mathfrak{B}_{D}$ is a Banach lattice and $\mathfrak{b}$ is linear and order preserving.

Proof The Bochner integrable functions form a Riesz space because of the inequality $\||x|-|y|\| \leq\|x-y\|$.

Theorem 4.6 Let $D$ be an ordered Banach space for which $D^{+}$is closed. Then $\mathfrak{b}$ is order preserving.

Proof Let $f \in \mathfrak{B}$ and $f \geq 0$. Then

$$
\alpha(\mathfrak{b}(f))=\int \alpha \circ f \mathrm{~d} \mu \geq 0 \quad\left(\alpha \in\left(D^{\prime}\right)^{+}\right) .
$$

Whence $\mathfrak{b}(f) \geq 0$, by Theorem 3.2.

\subsection{For the rest of this section, $D$ is an ordered Banach space.}

4.8 The following is a list of results presented in 4.9-4.16.

(a) $\mathfrak{B}^{+}$is closed if and only if $D^{+}$is (4.9).

(b) If $\mathfrak{B}$ is directed, then so is $D$ (straightforward, see also Theorem 4.12).

(c) Let $C>0$ and $D^{+}$be closed and generating. If $\|\cdot\|$ is $C$-absolutely dominating, then so is $\|\cdot\|_{\mathfrak{B}}$ (Lemma 4.11).

(d) Let $C>0$. If $\mathfrak{B}$ is directed and $\|\cdot\|_{\mathfrak{B}}$ is $C$-absolutely dominating, then so is $\|\cdot\|$ (Theorem 4.12).

(e) $\mathfrak{B}^{+}$is closed and generating if and only if $D^{+}$is closed and generating (Theorem 4.16).

(f) If there exist disjoint $A_{1}, A_{2}, \ldots$ in $\mathcal{A}$ with $0<\mu(A)<\infty$ for all $n$ : If $\mathfrak{B}^{+}$is generating, then so is $D^{+}$and $\|\cdot\|$ is absolutely dominating (Corollary 4.14).

(g) If no such $A_{1}, A_{2}, \ldots$ exist: $\mathfrak{B}^{+}$is generating if and only if $D^{+}$is generating (4.15).

4.9 Whenever $f \in \mathfrak{B}, f_{n} \in \mathfrak{B}^{+}$with $\int\left\|f-f_{n}\right\| \mathrm{d} \mu \rightarrow 0$, then there exist Bochner integrable $g_{n} \geq 0$ with $\sum_{n \in \mathbb{N}} \int\left\|f-g_{n}\right\| \mathrm{d} \mu<\infty$; this implies $g_{n} \rightarrow f \mu$-almost everywhere. So whenever $D^{+}$is closed this implies $f \geq 0 \mu$-almost everywhere.

We infer that $\mathfrak{B}^{+}$is closed whenever $D^{+}$is.

On the other hand, if $\mathfrak{B}^{+}$is closed then so is $D^{+}$. Indeed, let $A \in \mathcal{A}, 0<\mu(A)<\infty$. If $a_{n} \in D^{+}$and $a_{n} \rightarrow a$, then $\left\|a \mathbb{1}_{A}-a_{n} \mathbb{1}_{A}\right\|_{\mathfrak{B}} \rightarrow 0$. Therefore $a \in D^{+}$. 
Lemma 4.10 Suppose $D^{+}$is generating and $C>0$ is such that $\|\cdot\|$ is $C$-absolutely dominating. Let $f: X \rightarrow D$ be simple, let $\varepsilon>0$. Then there exists a simple $g: X \rightarrow$ $D^{+}$with $-g \leq f \leq g$ and $\int\|g\| \mathrm{d} \mu \leq C \int\|f\| \mathrm{d} \mu+\varepsilon$.

Proof Write $f=\sum_{n=1}^{N} x_{n} \mathbb{1}_{A_{n}}$ with disjoint sets $A_{1}, \ldots, A_{N} \in \mathcal{A}$ of finite measure and $x_{1}, \ldots, x_{N} \in D$. Let $\kappa=\mu\left(\bigcup_{n=1}^{N} A_{n}\right)$ and assume $\kappa>0$. For each $n$, choose $a_{n} \in D^{+},-a_{n} \leq x_{n} \leq a_{n},\left\|a_{n}\right\| \leq C\left\|x_{n}\right\|+\frac{\varepsilon}{\kappa}$. Put $g=\sum_{n=1}^{N} a_{n} \mathbb{1}_{A_{n}}$. Then $-g \leq f \leq g$ and $\int\|g\| \mathrm{d} \mu=\sum_{n=1}^{N}\left\|a_{n}\right\| \mu\left(A_{n}\right) \leq \sum_{n=1}^{N}\left(C\left\|x_{n}\right\|+\frac{\varepsilon}{\kappa}\right) \mu\left(A_{n}\right)=$ $C \int\|f\| \mathrm{d} \mu+\varepsilon$.

Lemma 4.11 Suppose $D^{+}$is closed and generating and $C>0$. Then $\mathfrak{B}^{+}$is closed and generating. If $\|\cdot\|$ is $C$-absolutely dominating, then so is $\|\cdot\|_{\mathfrak{B} \text {. }}$

Proof $\mathfrak{B}^{+}$is closed by 4.9. Assume that $\|\cdot\|$ is $C$-absolutely dominating. Let $f: X \rightarrow$ $D$ be Bochner integrable and let $\varepsilon>0$. We prove there exists a Bochner integrable $g: X \rightarrow D^{+}$with $-g \leq f \leq g \mu$-a.e. and $\int\|g\| \mathrm{d} \mu \leq C\left(\int\|f\| \mathrm{d} \mu+\varepsilon\right)$. Choose simple functions $s_{1}, s_{2}, \ldots$ with $\int\left\|f-s_{n}\right\| \mathrm{d} \mu<\varepsilon 2^{-n-1}$. Then $s_{n} \rightarrow f$ pointwise outside a $\mu$-null set $Y$.

Define $f_{1}=s_{1}, f_{n}=s_{n}-s_{n-1}$ for $n \in\{2,3, \ldots\}$. Observe

$$
\begin{aligned}
& \int\left\|f_{1}\right\| \mathrm{d} \mu<\int\|f\| \mathrm{d} \mu+\varepsilon 2^{-2}, \\
& \int\left\|f_{n}\right\| \mathrm{d} \mu<\varepsilon 2^{-n-1}+\varepsilon 2^{-n-2}<\varepsilon 2^{-n} \quad(n \in\{2,3, \ldots\}) .
\end{aligned}
$$

For each $n$, choose a simple $g_{n}: X \rightarrow D^{+}$with $-g_{n} \leq f_{n} \leq g_{n}$ such that

$$
\begin{aligned}
& \int\left\|g_{1}\right\| \mathrm{d} \mu<C\left(\int\|f\| \mathrm{d} \mu+\varepsilon 2^{-2}\right), \\
& \int\left\|g_{n}\right\| \mathrm{d} \mu<C \varepsilon 2^{-n} \quad(n \in\{2,3, \ldots\}) .
\end{aligned}
$$

As $\sum_{n \in \mathbb{N}} \int\left\|g_{n}\right\| \mathrm{d} \mu<\infty$ there is a $\mu$-null set $Z \subset X$ for which

$$
\sum_{n \in \mathbb{N}}\left\|g_{n}(x)\right\|<\infty \quad(x \in X \backslash Z) .
$$

Put $X_{0}:=X \backslash(Y \cup Z)$. Define $g: X \rightarrow D^{+}$by

$$
g(x)= \begin{cases}\sum_{n \in \mathbb{N}} g_{n}(x) & x \in X_{0}, \\ 0 & x \notin X_{0} .\end{cases}
$$

Then $g$ is Bochner integrable and $\int\|g\| \mathrm{d} \mu \leq \sum_{n \in \mathbb{N}} \int\left\|g_{n}\right\| \mathrm{d} \mu \leq C\left(\int\|f\| \mathrm{d} \mu+\varepsilon\right)$.

Moreover, for $x \in X_{0}$ we have $-g(x) \leq \sum_{n=1}^{N} f_{n}(x)=s_{N}(x) \leq g(x)$ for all $N$, whereas $s_{N}(x) \rightarrow f(x)$ since $x \notin Y$. From the closedness of $D^{+}$it follows that $-g \leq f \leq g$ on $X_{0}$. 
In the following Theorems $(4.12,4.13,4.14$ and 4.15) we derive properties of $D$ from properties of $\mathfrak{B}$. In Theorem 4.16 we show that $D$ has a closed and generating cone if and only if $\mathfrak{B}$ does.

Theorem 4.12 Assume $\mathfrak{B}$ is directed. Let $C>0$ and suppose $\|\cdot\|_{\mathfrak{B}}$ is $C$-absolutely dominating. Then $D$ is directed and $\|\cdot\|$ is $C$-absolutely dominating.

Proof Let $x \in D$ and $x \neq 0$. Let $A \in \mathcal{A}$ with $0<\mu(A)<\infty$. Then $x \mathbb{1}_{A} \in \mathfrak{B}$, $\left\|x \mathbb{1}_{A}\right\|_{\mathfrak{B}}=\mu(A)\|x\|$. Let $C^{\prime}>C$. There is a $g \in \mathfrak{B}$ with $-g \leq x \mathbb{1}_{A} \leq g$, $\int\|g(t)\| \mathrm{d} \mu(t) \leq C^{\prime}\left\|x \mathbb{1}_{A}\right\|_{\mathfrak{B}}$. Then $\int_{A}\|g(t)\| \mathrm{d} \mu(t) \leq C^{\prime} \mu(A)\|x\|$, so $\mu\{t \in A$ : $\left.\|g(t)\|>C^{\prime}\|x\|\right\}<\mu(A)$. In particular, there is a $t \in A$ with $\|g(t)\| \leq C^{\prime}\|x\|$ and $-g(t) \leq x \leq g(t)$.

Theorem 4.13 Let $D$ be an ordered Banach space such that the Bochner integrable functions $\mathbb{N} \rightarrow D$ form a directed space. Then $D$ is directed and $\|\cdot\|$ is absolutely dominating.

Proof $D$ is directed. In case $\|\cdot\|$ is not absolutely dominating, there exist $x_{1}, x_{2}, \ldots \in$ $D$ such that for every $n$

$$
2^{n}\left\|x_{n}\right\| \leq \inf \left\{\|a\|: a \in D^{+},-a \leq x_{n} \leq a\right\}
$$

and $\left\|x_{n}\right\|=2^{-n}$. Then $n \mapsto x_{n}$ is Bochner integrable, so, by our assumption, there exist $a_{n} \in D^{+}$with $-a_{n} \leq x_{n} \leq a_{n}$ for all $n$ and $\sum_{n \in \mathbb{N}}\left\|a_{n}\right\|<\infty$ which is false.

Corollary 4.14 Suppose there exist disjoint $A_{1}, A_{2}, \ldots$ in $\mathcal{A}$ with $0<\mu\left(A_{n}\right)<\infty$ for all $n$. Suppose $\mathfrak{B}$ is directed. Then $D$ is directed and $\|\cdot\|$ is absolutely dominating.

Proof This follows from Theorem 4.13 since $f \mapsto \sum_{n \in \mathbb{N}} f(n) \mathbb{1}_{A_{n}}$ forms an isometric order preserving isomorphism from the Bochner integrable functions $\mathbb{N} \rightarrow D$ into $\mathfrak{B}$.

4.15 Whenever there do not exist $A_{1}, A_{2}, \ldots$ as in Corollary 4.14, then $X=A_{1} \cup$ $\cdots \cup A_{N}$, where $A_{1}, \ldots, A_{N}$ are disjoint atoms. Let $\alpha_{n}=\mu\left(A_{n}\right) \in(0, \infty)$. Define a norm $\|\cdot\|_{N}$ on $D^{N}$ by $\|x\|_{N}=\sum_{n=1}^{N}\left\|x_{n}\right\|$. Then $T: \mathfrak{B} \rightarrow D^{N}$ defined by $T\left(\sum_{n=1}^{N} x_{n} \mathbb{1}_{A_{n}}\right)=\left(\alpha_{1} x_{1}, \ldots, \alpha_{N} x_{N}\right)$, is an isometric order preserving isomorphism. Therefore,

$$
D^{+} \text {is generating } \Longleftrightarrow\left(D^{N}\right)^{+} \text {is generating } \Longleftrightarrow \mathfrak{B}^{+} \text {is generating. }
$$

Theorem 4.16 $D^{+}$is closed and generating if and only if $\mathfrak{B}^{+}$is. Moreover, if $D^{+}$is closed and generating and $C>0$, then $\|\cdot\|$ is $C$-absolutely dominating if and only if $\|\cdot\|_{\mathfrak{B}}$ is.

Proof This follows from Lemma 4.11 and Theorem 4.12. 


\section{An extension of the Bochner integral}

We present the definition of a Banach cover (Definition 5.2), some examples, and use this notion to extend the Bochner integral to functions with values in such a vector space (5.8).

The next result follows by definition of the Bochner integral or by 4.4(c).

Theorem 5.1 Let $D_{1}$ and $D$ be Banach spaces and suppose $D_{1} \subset D$ and the inclusion map is continuous. Suppose $f: X \rightarrow D$ has values in $D_{1}$ and is Bochner integrable as map $X \rightarrow D_{1}$ with integral I. Then $f$ is Bochner integrable as a map $X \rightarrow D$ with integral $I$.

Definition 5.2 Let $E$ be a vector space. Suppose $\mathbb{B}$ is a collection of Banach spaces whose underlying vector spaces are linear subspaces of $E . \mathbb{B}$ is called a Banach cover of $E$ if $\bigcup \mathbb{B}=E$ and for all $D_{1}$ and $D_{2}$ in $\mathbb{B}$ there exists a $D \in \mathbb{B}$ with $D_{1}, D_{2} \subset D$ such that both inclusion maps $D_{1} \rightarrow D$ and $D_{2} \rightarrow D$ are continuous.

For a $D \in \mathbb{B}$, we write $\|\cdot\|_{D}$ for its norm if not indicated otherwise.

If $E$ is an ordered vector space, then an ordered Banach cover is a Banach cover whose elements are seen as ordered subspaces of $E$.

5.3 A bit of pedantry: strictly speaking, a Banach space is a couple $(D,\|\cdot\|)$ consisting of a vector space $D$ and a norm $\|\cdot\|$. One usually talks about the "Banach space $D$ ", the norm being understood. Mostly, we adopt that convention but not always. In the context of the above definitions one has to be careful. A Banach cover may contain several Banach spaces with the same underlying vector space, so that a formula like " $D_{1}, D_{2} \subset D$ " really is ambiguous. What we mean is only an inclusion relation between the vector spaces and no connection between the norms is assumed a proiri.

However, suppose $\left(D_{1},\|\cdot\|_{1}\right)$ and $\left(D_{2},\|\cdot\|_{2}\right)$ are elements of a Banach cover $\mathbb{B}$ and $D_{1}=D_{2}$. There is a Banach space $\left(D,\|\cdot\|_{D}\right)$ in $\mathbb{B}$ with $D_{1}, D_{2} \subset D$ and with continuous inclusion maps. If a sequence $\left(x_{n}\right)_{n \in \mathbb{N}}$ in $D_{1}\left(=D_{2}\right)$ is $\|\cdot\|_{1}$-convergent to $a$ and $\|\cdot\|_{2}$-convergent to $b$, then it is $\|\cdot\|_{D}$-convergent to $a$ and $b$, so $a=b$. Hence, by the Closed Graph Theorem the norms $\|\cdot\|_{1}$ and $\|\cdot\|_{2}$ are equivalent.

Similarly, if $\left(D_{1},\|\cdot\|_{1}\right)$ and $\left(D_{2},\|\cdot\|_{2}\right)$ are elements of a Banach cover and $D_{1} \subset D_{2}$, then the inclusion map $D_{1} \rightarrow D_{2}$ automatically is $\|\cdot\|_{1}-\|\cdot\|_{2}$-continuous.

Example 5.4 In [18], Thomas considers quasicomplete Hausdorff locally convex vector spaces. For every bounded closed absolutely convex subset $B$ of such a space $E$ its linear hull $E_{B}$ is a Banach space under a norm whose closed unit ball is $B$. If $B_{1}$ and $B_{2}$ are bounded closed absolutely convex sets, then so is the closure of $B_{1}+B_{2}$. It follows that the spaces $E_{B}$ form a Banach cover of $E$.

Example 5.5 Let $E$ be a uniformly complete Riesz space. The set of principal ideals $\mathbb{B}=\left\{\left(E_{u},\|\cdot\|_{u}\right): u \in E^{+}, u \neq 0\right\}$ is an ordered Banach cover of $E$ : for $u, v \in E^{+}$ with $u, v>0$ one has $E_{u}, E_{v} \subset E_{u+v}$ and $\|\cdot\|_{u+v} \leq\|\cdot\|_{u}$ on $E_{u}$.

Example 5.6 Let $(Y, \mathcal{B}, v)$ be a complete $\sigma$-finite measure space. Let $M$ be the space of classes of measurable functions $Y \rightarrow \mathbb{R}$. A function $\rho: M \rightarrow[0, \infty]$ is called an function norm if (i) $\rho(f)=0 \Longleftrightarrow f=0$ a.e., (ii) $\rho(\alpha f)=|\alpha| \rho(f)$ (where 
$0 \cdot \infty=0$ ), (iii) $\rho(|f|)=\rho(f)$, (iv) $\rho(f+g) \leq \rho(f)+\rho(g)$, (v) $0 \leq f \leq g$ a.e. implies $\rho(f) \leq \rho(g)$ for $f, g \in M$ and $\alpha \in \mathbb{R}$. For such function norm $\rho$ the set

$$
L_{\rho}=\{f \in M: \rho(f)<\infty\}
$$

is a normed Riesz space called a Köthe space (see [11, Ch. III §18] or [12, Ch. 1 §9]).

A Köthe space $L_{\rho}$ is complete, i.e., a Banach lattice if and only if for all $u_{n} \in L_{\rho}^{+}$ with $\sum_{n \in \mathbb{N}} \rho\left(u_{n}\right)<\infty, \sum_{n \in \mathbb{N}} u_{n}$ is an element of $L_{\rho}$ ([11, Theorem 19.3]). Examples of complete Köthe spaces are Orlicz spaces $([11, \S 20])$. In particular, the Banach spaces $L^{p}(v)$ for $p \geq 1$ are Köthe spaces. We introduce other examples:

For $w \in M^{+}$with $w>0$ a.e. define $\rho_{w}: M \rightarrow[0, \infty]$ by

$$
\rho_{w}(f):=\int|f| w \mathrm{~d} v .
$$

Then $\rho_{w}$ is a function norm. Both the set $\left\{L_{\rho_{w}}: w \in M^{+}, w>0\right.$ a.e. $\}$ and the set of all complete Köthe spaces are Banach covers of $M$ (see Appendix 1).

Example 5.7 (See Example 5.4.) In the situation of [18], the functions in our set $\mathfrak{U}$ are precisely the functions called "totally summable" by Thomas. Apparently, the totally summable functions form a vector space, and the integral of such a function in the sense of 5.8 equals its Pettis integral as considered by Thomas.

5.8 Let $E$ be a vector space with a Banach cover $\mathbb{B}$. Let $(X, \mathcal{A}, \mu)$ be a complete $\sigma$-finite measure space, $\mu \neq 0$.

(1) For $D \subset \mathbb{B}$ denote by $B_{D}$ the vector space of all Bochner integrable functions $X \rightarrow D$, and, by $b_{D}$ the Bochner integral $B_{D} \rightarrow D$.

(2) Let $D_{1}, D_{2} \in \mathbb{B}, f_{1} \in B_{D_{1}}, f_{2} \in B_{D_{2}}, f_{1}=f_{2} \mu$-a.e. Then $b_{D_{1}}\left(f_{1}\right)=b_{D_{2}}\left(f_{2}\right)$. Proof. Choose $D \in \mathbb{B}$ as in Definition 5.2. Then $f_{1}, f_{2} \in B_{D}$ and $b_{D_{1}}\left(f_{1}\right)=$ $b_{D}\left(f_{1}\right)=b_{D}\left(f_{2}\right)=b_{D_{2}}\left(f_{2}\right)$.

(3) If $D_{1}, D_{2} \in \mathbb{B}$ have the same underlying vector space, then $B_{D_{1}}=B_{D_{2}}$ since the identity map $D_{1} \rightarrow D_{2}$ is a homeomorphism (see 5.3).

(4) We call a function $f: X \rightarrow E \mathbb{B}$-integrable if there is a $D \in \mathbb{B}$ such that $f$ is $\mu$-a.e. equal to some element of $B_{D}$.

(5) By $\mathfrak{U}$ we indicate the vector space of all $\mu$-equivalence classes of $\mathbb{B}$-integrable functions.

For $D \in \mathbb{B}$ we have a natural map $T_{D}: B_{D} \rightarrow \mathfrak{U}$, assigning to every element of $B_{D}$ its $\mu$-equivalence class. The space $T_{D}\left(B_{D}\right)$ is a Banach space, which we indicate by $\mathfrak{B}_{D} .{ }^{6}$ We write $\mathfrak{b}_{D}$ for the map $\mathfrak{B}_{D} \rightarrow D$ determined by

$$
\mathfrak{b}_{D}\left(T_{D}(f)\right)=b_{D}(f) \quad\left(f \in B_{D}\right) .
$$

$\mathfrak{U}$ is the union of the sets $\mathfrak{B}_{D}$. By (2) there is a unique $\mathfrak{u}: \mathfrak{U} \rightarrow E$ determined by

$$
\mathfrak{u}(f)=\mathfrak{b}_{D}(f) \quad\left(D \in \mathbb{B}, f \in \mathfrak{B}_{D}\right) .
$$

\footnotetext{
${ }^{6}$ Even though we use the same notation as in Sect. 4, see 4.3, the meaning of $\mathfrak{B}_{D}$ is slightly different.
} 
The above leads to the following theorem.

Theorem $5.9 \mathfrak{U}$ is a vector space, $\mathfrak{u}$ is linear and $\left\{\mathfrak{B}_{D}: D \in \mathbb{B}\right\}$ is a Banach cover of $\mathfrak{U}$.

$\mathbf{5 . 1 0}$ (1) For any vector space the finite dimensional linear subspaces form a Banach cover.

(2) If $E$ is a Banach space, then $\{E\}$ is a Banach cover.

(3) If $E$ is a Banach space and $\mathbb{B}$ is a Banach cover with $E \in \mathbb{B}$, then $\mathfrak{U}$ is just the space of (classes of) Bochner integrable functions $X \rightarrow E$ and $\mathfrak{u}$ is the Bochner integral.

(4) A special case of (3): Let $E$ be a uniformly complete Riesz space with a unit $e$ and let $\mathbb{B}$ be the Banach cover of principal ideals as in Example 5.5. Then $\left(E,\|\cdot\|_{e}\right) \in \mathbb{B}$.

5.11 Let $E$ be a vector space and $\mathbb{B}_{1}$ and $\mathbb{B}_{2}$ be Banach covers of $E$. Suppose that for all $D_{1} \in \mathbb{B}_{1}$ there exists a $D_{2} \in \mathbb{B}_{2}$ with $D_{1} \subset D_{2}$ such that the inclusion map is continuous. Write $\mathfrak{U}_{i}, \mathfrak{u}_{i}$ for the set of $\mathbb{B}_{i}$-integrable functions and the $\mathbb{B}_{i}$-integral, for $i \in\{1,2\}$. Then $\mathfrak{U}_{1} \subset \mathfrak{U}_{2}$ and $\mathfrak{u}_{1}=\mathfrak{u}_{2}$ on $\mathfrak{U}_{1}$.

Example 5.12 (Different covers and different integrals) Let $(E,\|\cdot\|)$ be an infinite dimensional Banach space. Let $T: E \rightarrow E$ a linear bijection that is not continuous; say, there exist $x_{n} \in E$ with $\left\|x_{n}\right\|=2^{-n}, T x_{n}=x_{n}, T\left(\sum_{n \in \mathbb{N}} x_{n}\right) \neq \sum_{n \in \mathbb{N}} x_{n}$. Define $\|x\|_{T}=\|T x\|$ for $x \in E$. Then $\left(E,\|\cdot\|_{T}\right)$ is a Banach space. The map $f: \mathbb{N} \rightarrow E$ given by $f(n)=x_{n}$ is Bochner integrable in $(E,\|\cdot\|)$ and in $\left(E,\|\cdot\|_{T}\right)$, but the integrals do not agree. Whence with $\mathbb{B}_{1}=\{(E,\|\cdot\|)\}$ and $\mathbb{B}_{2}=\left\{\left(E,\|\cdot\|_{T}\right)\right\}$ we have $f \in \mathfrak{U}_{1} \cap \mathfrak{U}_{2}$ but $\mathfrak{u}_{1}(f) \neq \mathfrak{u}_{2}(f)$.

As an immediate consequence of Theorem 4.5 we obtain the following theorem.

Theorem 5.13 Suppose E is a Riesz space and $\mathbb{B}$ is a Banach cover of $E$ that consists of Banach lattices that are Riesz subspaces of E. Then $\mathfrak{U}$ is a Riesz space and $\mathfrak{u}$ is order preserving.

Proof This is a consequence of Theorem 4.5.

5.14 Whenever $E$ is an ordered vector space and $\mathbb{B}$ an ordered Banach cover of $E$, then $\mathfrak{U}$ is an ordered vector space. In order for $\mathfrak{u}$ to be order preserving, one needs a condition on $\mathbb{B}$. This and other matters will be treated in Sect. 7. A sufficient condition turns out to be closedness of $D^{+}$for every $D \in \mathbb{B}$ (see Theorems 4.6 and 7.1). First we will see in Sect. 6 that all Archimedean directed ordered vector spaces admit such ordered Banach covers. (The Archimedean property is necessary as follows easily from Theorem 3.2).

5.15 Whenever $E$ is a vector space and $\mathbb{B}$ is a Banach cover of $E$, then the set

$$
\{A \subset E \text { : there exists a } D \in \mathbb{B} \text { such that } A \text { is bounded in } D\}
$$

forms a bornology on $E$ (we refer to the book of Hogbe-Nlend [10] for the theory of bornologies). 


\section{Covers of ordered Banach spaces with closed generating cones}

In this section $E$ is an Archimedean directed ordered vector space. $\mathbb{B}$ is the collection of all ordered Banach spaces that are ordered linear subspaces of $E$ whose cones are closed and generating.

We intend to prove that $\mathbb{B}$ is a Banach cover of $E$ (Theorem 6.5).

Lemma 6.1 Let $\left(D_{1},\|\cdot\|_{1}\right),\left(D_{2},\|\cdot\|_{2}\right)$ be in $\mathbb{B}$. Let $z_{1}, z_{2}, \ldots \in D_{1} \cap D_{2}, a \in D_{1}$, $b \in D_{2},\left\|z_{n}-a\right\|_{1} \rightarrow 0,\left\|z_{n}-b\right\|_{2} \rightarrow 0$. Then $a=b$.

Proof We may assume $\sum_{n \in \mathbb{N}}\left\|z_{n}-a\right\|_{1}<\infty$ and $\sum_{n \in \mathbb{N}}\left\|z_{n}-b\right\|_{2}<\infty$. Then $z_{n} \stackrel{u}{\rightarrow} a$ in $D_{1}$ and $z_{n} \stackrel{u}{\rightarrow} b$ in $D_{2}$ by Theorem 3.9. Then $z_{n} \stackrel{u}{\rightarrow} a$ and $z_{n} \stackrel{u}{\rightarrow} b$ in $E$. Because $E$ is Archimedean, $a=b$.

6.2 If $D$ is an ordered Banach space with closed generating cone $D^{+}$, under each of two norms $\|\cdot\|_{1}$ and $\|\cdot\|_{2}$, then these norms are equivalent. Indeed, the identity map $\left(D,\|\cdot\|_{1}\right) \rightarrow\left(D,\|\cdot\|_{2}\right)$ has a closed graph by Lemma 6.1 .

Theorem 6.3 Let $\left(D_{1},\|\cdot\|_{1}\right),\left(D_{2},\|\cdot\|_{2}\right)$ be in $\mathbb{B} . D_{1}+D_{2}$ is an ordered Banach space with closed generating cone under the norm $\|\cdot\|: D_{1}+D_{2} \rightarrow[0, \infty)$ defined by

$$
\|z\|:=\inf \left\{\|x\|_{1}+\|y\|_{2}: x \in D_{1}, y \in D_{2}, z=x+y\right\} .
$$

Moreover, if $C>0$ and $\|\cdot\|_{1}$ and $\|\cdot\|_{2}$ are $C$-absolutely dominating, then so is $\|\cdot\|$.

Proof $D_{1} \times D_{2}$ is a Banach space under the norm $(x, y) \mapsto\|x\|_{1}+\|y\|_{2}$. From Lemma 6.1 it follows that $\Delta:=\left\{(a, b) \in D_{1} \times D_{2}: a=-b\right\}$ is closed in $D_{1} \times D_{2}$. Then $D_{1} \times D_{2} / \Delta$ is a Banach space under the quotient norm. This means that $D_{1}+D_{2}$ is a Banach space under $\|\cdot\|$. (In particular, $\|\cdot\|$ is a norm.)

Since $D_{1}^{+}+D_{2}^{+} \subset\left(D_{1}+D_{2}\right)^{+}$, the latter is generating.

We prove that $\left(D_{1}+D_{2}\right)^{+}$is closed. Let $u_{1}, u_{2}, \ldots \in D_{1}+D_{2}, \sum_{n \in \mathbb{N}}\left\|u_{n}\right\|<\infty$; we prove $u_{n} \stackrel{u}{\rightarrow} 0$. Choose $x_{n} \in D_{1}, y_{n} \in D_{2}$ with $u_{n}=x_{n}+y_{n}$ and $\sum_{n \in \mathbb{N}}\left\|x_{n}\right\|_{1}<$ $\infty, \sum_{n \in \mathbb{N}}\left\|y_{n}\right\|_{2}<\infty$. Then, see Theorem 3.9, $x_{n} \stackrel{u}{\rightarrow} 0$ in $D_{1}$ and $y_{n} \stackrel{u}{\rightarrow} 0$ in $D_{2}$. It follows that $u_{n} \stackrel{u}{\rightarrow} 0$ in $D_{1}+D_{2}$. By Theorem 3.9 it follows that $\left(D_{1}+D_{2}\right)^{+}$is closed.

Suppose $C>0$ is such that $\|\cdot\|_{1}$ and $\|\cdot\|_{2}$ are $C$-absolutely dominating. Let $z \in$ $D_{1}+D_{2}, \varepsilon>0$. Choose $x \in D_{1}, y \in D_{2}$ with $z=x+y,\|x\|_{1}+\|y\|_{2} \leq\|z\|+\frac{\varepsilon}{3}$. Choose $a \in D_{1}^{+}$with $-a \leq x \leq a,\|a\|_{1}<C\|x\|_{1}+\frac{\varepsilon}{3}$ and $b \in D_{2}^{+}$with $-b \leq y \leq b$, $\|b\|_{2}<C\|y\|_{2}+\frac{\varepsilon}{3}$. Set $c=a+b$. Then $c \in\left(D_{1}+D_{2}\right)^{+},-c \leq z \leq c$ and $\|c\| \leq\|a\|_{1}+\|b\|_{2}<C\|x\|_{1}+C\|y\|_{2}+2 \frac{\varepsilon}{3}<C\|z\|+\varepsilon$.

6.4 Let $x \in E$. (We make a $D \in \mathbb{B}$ with $x \in D$.) Choose $a \in E$ such that $-a \leq x \leq a$. Let $D=\mathbb{R}(a-x)+\mathbb{R}(a+x)=\mathbb{R} a+\mathbb{R} x$. $D$ is a directed ordered vector space. Define $\|\cdot\|: D \rightarrow[0, \infty)$ by

$$
\|y\|:=\inf \{s \geq 0:-s a \leq y \leq s a\} .
$$


Then $\|\cdot\|$ is a norm on $D,-\|y\| a \leq y \leq\|y\| a$ for all $y \in D$ and $\|a\|=1$. Thus $(D,\|\cdot\|)$ is a directed ordered Banach space. Moreover $D^{+}$is closed: Let $y \in D$, $y_{1}, y_{2}, \cdots \in D^{+},\left\|y-y_{n}\right\|<\frac{1}{n}$. Then $y \geq y_{n}-\frac{1}{n} a \geq-\frac{1}{n} a$, so $y \geq 0$.

$\|\cdot\|$ is 1 -absolutely dominating: $\|a\|=1$, so $\inf \left\{\|c\|: c \in D^{+},-c \leq y \leq c\right\} \leq$ $\inf \{s \geq 0:-s a \leq y \leq s a\}=\|y\|$.

Even $\|y\|=\inf \left\{\|c\|: c \in D^{+},-c \leq y \leq c\right\}$ : For $c \in D^{+}$with $-c \leq y \leq c$ and $s \geq 0$ such that $c \leq s a$ we have $-s a \leq-c \leq y \leq c \leq s a$ and so $\|y\| \leq\|c\|$.

Theorem 6.5 $\mathbb{B}$ is a Banach cover of E. Moreover,

$$
\left\{D \in \mathbb{B}:\|\cdot\|_{D} \text { is } 1-\text { absolutely dominating }\right\}
$$

is a Banach cover of $E$.

Proof By 6.4 each element of $E$ is contained in an ordered Banach space with closed generating cone (with a 1-absolutely dominating norm). By Theorem 6.3 and by definition of the norm, $\mathbb{B}$ forms a Banach cover of $E$.

6.6 It is reasonable to ask if an analogue of Theorem 6.5 holds in the world of Riesz spaces: does every Archimedean Riesz space have a Banach cover consisting of Riesz spaces? The answer is negative.

Let $E$ be the Riesz space of all functions $f$ on $\mathbb{N}$ for which there exist $N \in \mathbb{N}$ and $r, s \in \mathbb{R}$ such that $f(n)=s n+r$ for $n \geq N$. Suppose $E$ has a Banach cover $\mathbb{B}$ consisting of Riesz subspaces of $E$. There is a $D \in \mathbb{B}$ that contains the constant function $\mathbb{1}$ and the identity map $i: \mathbb{N} \rightarrow \mathbb{N}$. For every $n \in \mathbb{N}$,

$$
\mathbb{1}_{\{1, \ldots, n\}}=\mathbb{1} \vee(n+1) \mathbb{1}-i \vee n \mathbb{1} \in D
$$

It follows that $D=E$, so $E$ is a Banach space under some norm.

But $E$ is the union of an increasing sequence $D_{1} \subset D_{2} \subset \cdots$ of finite dimensional-hence, closed-linear subspaces:

$$
D_{n}=\mathbb{R} \mathbb{1}+\mathbb{R} i+\mathbb{R}_{\{1\}}+\cdots+\mathbb{R} \mathbb{1}_{\{n\}} .
$$

By Baire's Category Theorem, some $D_{n}$ has nonempty interior in $E$. Then $E=D_{n}$ and we have a contradiction.

6.7 In Theorem 6.5 we single out one particular Banach cover $\mathbb{B}$. If we consider only Banach covers consisting of directed spaces with closed cones, this $\mathbb{B}$ is the largest and gives us the largest collection of integrable functions. Without directedness there may not be a largest Banach cover. For instance, consider Example 5.12. Impose on $E$ the trivial ordering $\left(x \leq y\right.$ if and only if $x=y$ ). Then $E^{+}=\{0\}$, and both $\mathbb{B}_{1}$ and $\mathbb{B}_{2}$ consist of Banach spaces with closed (but not generating) cones.

\section{The integral for an Archimedean ordered vector space}

As a consequence of Theorem 4.6 we obtain the following extension of Theorem 5.13. 
Theorem 7.1 Let $E$ be an ordered vector space with an ordered Banach cover $\mathbb{B}$ so that $D^{+}$is closed for all $D \in \mathbb{B}$. Then $\mathfrak{u}$ is order preserving. Moreover, $E$ and $\mathfrak{U}$ are Archimedean.

Lemma 7.2 Let $D$ be an ordered Banach space with a closed generating cone $D^{+}$. Let $T$ be a linear order preserving map of $D$ into an Archimedean ordered vector space $H$. Then $\operatorname{ker} T$ is closed and $T(D)$ equipped with the norm $\|\cdot\|_{q}$ given by

$$
\|z\|_{q}=\inf \{\|x\|: x \in D, T x=z\}
$$

has a closed generating cone $T(D)^{+}$.

Proof (I) Let $x_{1}, x_{2}, \ldots \in \operatorname{ker} T, x \in D, x_{n} \rightarrow x$; we prove $x \in \operatorname{ker} T$. We assume $\sum_{n \in \mathbb{N}}\left\|x-x_{n}\right\|<\infty$. By Theorem $3.9 x_{n} \stackrel{u}{\rightarrow} x$. By Lemma $3.8 T x_{n} \stackrel{u}{\rightarrow} T x$, so $T x=0$. (II) $D / \operatorname{ker} T$ is a Banach space under the quotient norm $\|\cdot\|_{Q}$. The formula $x+\operatorname{ker} T \mapsto T x$ describes a linear bijection $D / \operatorname{ker} T \rightarrow T(D)$ and

$$
\|x+\operatorname{ker} T\|_{Q}=\|T x\|_{q} \quad(x \in D) .
$$

It follows that $\|\cdot\|_{q}$ is indeed a norm, turning $T(D)$ into a Banach space.

$T\left(D^{+}\right) \subset T(D)^{+}$, whence $T(D)$ is directed.

We prove that $T(D)$ satisfies (iii) of Theorem 3.9: Let $z_{1}, z_{2}, \cdots \in T(D)$ be such that $\sum_{n \in \mathbb{N}}\left\|z_{n}\right\|_{q}<\infty$. Choose $x_{n} \in D$ such that $T x_{n}=z_{n}, \sum_{n \in \mathbb{N}}\left\|x_{n}\right\|<\infty$. Using (iii) for $D, x_{n} \stackrel{u}{\rightarrow} 0$. Then $z_{n}=T x_{n} \stackrel{u}{\rightarrow} 0$ by Lemma 3.8 .

7.3 In the proof of Lemma 7.2 we mentioned the inclusion $T\left(D^{+}\right) \subset T(D)^{+}$. This inclusion can be strict. Take $D=H=\mathbb{R}^{2}, T(x, y)=(x, x+y)$. Then $T\left(D^{+}\right) \neq$ $T(D)^{+}$.

From Theorems 3.11, 4.4(c) and Lemma 7.2 we get:

Theorem 7.4 Let $E_{1}, E_{2}$ be ordered vector spaces, $E_{i}$ endowed with the Banach cover $\mathbb{B}_{i}$ consisting of the ordered Banach spaces with closed generating cones. Let $T: E_{1} \rightarrow E_{2}$ be linear and order preserving. If $f: X \rightarrow E_{1}$ is $\mathbb{B}_{1}$-integrable, then $T \circ f: X \rightarrow E_{2}$ is $\mathbb{B}_{2}$-integrable, and $\mathfrak{u}_{2}(T \circ f)=T\left(\mathfrak{u}_{1}(f)\right)$.

7.5 In view of Theorem 3.11 the reader may wonder why in Theorem 7.4 T is required to be order preserving and not just order bounded, the more so because of the following considerations. Let $D$ and $H$ be as in Lemma 7.2 and $T$ be a linear order bounded map of $D$ into $H$. As the implication (c) $\Longrightarrow$ (a) of Theorem 3.9 is valid for Archimedean (but not necessarily directed) $D$, following the lines of the proof of Lemma 7.2 $\operatorname{ker} T$ is closed and $T(D)$ equipped with the norm as in (29) has a closed cone $T(D)^{+}$. However, we also need $T(D)$ to be directed and order boundedness of $T$ is no guarantee for that.

An alternative approach might be to drop the directedness condition on the spaces that constitute $\mathbb{B}$. However, the ordered Banach spaces with closed cones may not form a Banach cover.

For an example, let $E$ be $\ell^{\infty}$ and let $\mathbb{B}$ be the collection of all ordered Banach spaces that are subspaces of $\ell^{\infty}$ and have closed cones. We make $D_{1}, D_{2} \in \mathbb{B}$. For 
$D_{1}$ we take $\ell^{\infty}$ with the usual norm $\|\cdot\|_{\infty}$. Choose a linear bijection $T: \ell^{\infty} \rightarrow \ell^{\infty}$ that is not continuous. For $a \in \ell^{\infty}$ put $a^{\prime}=\left(a_{1},-a_{1}, a_{2},-a_{2}, \ldots\right)$. For $D_{2}$ we take the vector space $\left\{a^{\prime}: a \in \ell^{\infty}\right\}$ with the norm $\|\cdot\|_{T}$ given by $\left\|a^{\prime}\right\|_{T}=\|T a\|_{\infty}$. Then $D_{2}$ is a Banach space and $D_{2}^{+}$, begin $\{0\}$, is closed in $D_{2}$. Suppose $\mathbb{B}$ is a Banach cover. Let $D$ be as in Definition 5.2. By the Open Mapping Theorem the identity map $D_{1} \rightarrow D$ is a homeomorphism. By the continuity of the inclusion map $D_{2} \rightarrow D$ there exists a number $c$ such that $\left\|a^{\prime}\right\|_{T} \leq c\left\|a^{\prime}\right\|_{\infty}$ for all $a \in \ell^{\infty}$. Then $\|T a\|_{\infty} \leq c\left\|a^{\prime}\right\|_{\infty} \leq c\|a\|_{\infty}$ for $a \in \ell^{\infty}$, so $T$ is continuous. Contradiction.

Theorem 7.6 Let $E$ be an ordered vector space such that $E^{\sim}$ separates the points of $E .^{7}$ Assume $\mathbb{B}$ is a Banach cover consisting of ordered Banach spaces with closed generating cones. Let $f \in \mathfrak{U}$. Then $\alpha \circ f \in \mathcal{L}^{1}(\mu)$ for all $\alpha \in E^{\sim}$. Moreover, $I \in E$ is such that

$$
\alpha(I)=\int \alpha \circ f \mathrm{~d} \mu \text { for all } \alpha \in E^{\sim}
$$

if and only if $I=\mathfrak{u}(f)$.

Proof Let $f \in \mathfrak{U}$ and let $I=\mathfrak{u}(f)$. By Theorem $7.4 \alpha \circ f \in \mathcal{L}^{1}(\mu)$ for all $\alpha \in E^{\sim}$ and (31) holds. $I=\mathfrak{u}(f)$ is the only element of $E$ for which (31) holds because $E^{\sim}$ separates the points of $E$.

Remark 7.7 Functions with values in a Banach space that are Bochner integrable are also Pettis integrable. To some extent the statement of Theorem 7.6 is similar. Indeed, the definition of Pettis integrability could be generalised for vector spaces $V$ which are equipped with a set $S$ of linear maps $V \rightarrow \mathbb{R}$ that separates the points of $V$, in the sense that one calls a function $f: X \rightarrow V$ Pettis integrable if $\alpha \circ f \in \mathcal{L}^{1}(\mu)$ for all $\alpha \in S$ and there exists a $I \in V$ such that $\alpha(I)=\int \alpha \circ f \mathrm{~d} \mu$ for all $\alpha \in S$. Then Theorem 7.6 implies that every $f \in \mathfrak{U}$ is Pettis integrable when considering $V=E$ and $S=E^{\sim}$. Observe, however, that even for a Riesz space $E, E^{\sim}$ may be trivial (see, e.g., [11, 5.A]).

Theorem 7.8 Let $E$ be an ordered quasicomplete Hausdorff locally convex vector space with a closed generating cone. Let $\mathbb{B}_{1}$ be the Banach cover generated by the bounded closed absolutely convex subsets of $E$ (see Example 5.4). Let $\mathbb{B}_{2}$ be the Banach cover of ordered Banach spaces with closed generating cones (see Section 6 , $E$ is Archimedean by Theorem 3.2). Let $\mathfrak{U}_{i}$ and $\mathfrak{u}_{i}$ denote the $\mathbb{B}_{i}$-integrable functions and $\mathbb{B}_{i}$-integral, respectively. Then $\mathfrak{u}_{1}=\mathfrak{u}_{2}$ on $\mathfrak{U}_{1} \cap \mathfrak{U}_{2}$.

Proof If $f \in \mathfrak{U}_{1}$, then $f$ is Pettis integrable in the sense of Thomas [18, Definition 1] (or in Remark 7.7 with $V=E$ and $S=E^{\prime}$ ). By Theorem 7.6, for $f \in \mathfrak{U}_{1} \cap \mathfrak{U}_{2}$

$$
\alpha\left(\mathfrak{u}_{1}(f)\right)=\int \alpha \circ f \mathrm{~d} \mu=\alpha\left(\mathfrak{u}_{2}(f)\right) \quad\left(\alpha \in\left(E^{\prime}\right)^{+}\right),
$$

implying $\mathfrak{u}_{1}(f)=\mathfrak{u}_{2}(f)$ by Theorem 3.2.

\footnotetext{
7 We write $E^{\sim}$ for the space of order bounded linear maps $E \rightarrow \mathbb{R}$.
} 


\section{Comparison with other integrals}

In this section $(X, \mathcal{A}, \mu)$ is a complete $\sigma$-finite measure space and $E$ is a directed ordered vector space with an ordered Banach cover $\mathbb{B}$ so that $D^{+}$is closed for each $D \in \mathbb{B}$.

In 5.8 we have introduced an integral $\mathfrak{u}$ on a space $\mathfrak{U}$ of $\mathbb{B}$-integrable functions $X \rightarrow$ $E{ }^{8}$ In [16], starting from a natural integral $\varphi$ on the space $S$ of all simple functions $X \rightarrow E$ we have made integrals $\varphi_{V}, \varphi_{L}, \varphi_{L V}, \ldots$ on spaces $S_{V}, S_{L}, S_{L V}, \ldots$

There is an elementary connection: $S$ is part of $\mathfrak{U}$ and $\mathfrak{u}$ coincides with $\varphi$ on $S$. (Indeed, let $f \in S$. Being a finite set, $f(X)$ is contained in $D$ for some $D \in \mathbb{B}$. Then $f$ is Bochner integrable as a map $X \rightarrow D$.)

In general, $S_{V}$ and $S_{L}$ are not subsets of $\mathfrak{U}$, but we can prove that $\mathfrak{u}$ coincides with $\varphi_{V}$ on $S_{V} \cap \mathfrak{U}$ and with $\varphi_{L}$ on $S_{L} \cap \mathfrak{U}$. Better than that: $\mathfrak{u}$ is "compatible" with $\varphi_{V}$ in the sense that $\mathfrak{u}$ and $\varphi_{V}$ have a common order preserving linear extension $S_{V}+\mathfrak{U} \rightarrow E$. Similarly, $\mathfrak{u}$ is "compatible with $\varphi_{L}, \varphi_{L V}, \ldots$ ".

Lemma 8.1 (a) Let $f \in \mathfrak{U}, g \in S_{V}, f \leq g$. Then $\mathfrak{u}(f) \leq \varphi_{V}(g)$.

(b) Let $f \in \mathfrak{U}, g \in S_{L}, f \leq g$. Then $\mathfrak{u}(f) \leq \varphi_{L}(g)$.

Proof (a) By the definition of $\varphi_{V}$ and by the text preceding this lemma we have $\varphi_{V}(g)=\inf \{\varphi(h): h \in S, h \geq g\}=\inf \{\mathfrak{u}(h): h \in S, h \geq g\}$. As $g \geq f$ and $\mathfrak{u}$ is order preserving (Theorem 7.1), it follows that $\varphi_{V}(g) \geq \mathfrak{u}(f)$.

(b) Let $g \in S_{L}$ and assume $f \leq g$. Let $g_{1}, g_{2} \in S_{L}^{+}$be such that $g=g_{1}-g_{2}$. Let $\left(B_{i}\right)_{i \in \mathbb{N}}$ be a $\varphi$-partition for both $g_{1}$ and $g_{2}$. Write $A_{n}=\bigcup_{i=1}^{n} B_{i}$ for $n \in \mathbb{N}$. Then $f \mathbb{1}_{A_{n}} \leq g \mathbb{1}_{A_{n}}$, thus by (a) (and Theorem 7.1)

$$
\begin{aligned}
\mathfrak{u}\left(f \mathbb{1}_{A_{n}}\right) & \leq \mathfrak{u}\left(g \mathbb{1}_{A_{n}}\right)=\varphi\left(g \mathbb{1}_{A_{n}}\right)=\varphi\left(g_{1} \mathbb{1}_{A_{n}}\right)-\varphi\left(g_{2} \mathbb{1}_{A_{n}}\right) \\
& \leq \varphi_{L}\left(g_{1}\right)-\varphi\left(g_{2} \mathbb{1}_{A_{k}}\right) \quad(k \in \mathbb{N}, k<n) .
\end{aligned}
$$

Which implies $\mathfrak{u}\left(f \mathbb{1}_{A_{n}}\right)+\varphi\left(g_{2} \mathbb{1}_{A_{k}}\right) \leq \varphi_{L}\left(g_{1}\right)$ for all $k<n$. Then letting $n$ tend to $\infty$ (apply 4.4(d): $f(x) \mathbb{1}_{A_{n}}(x) \rightarrow f(x)$ for all $x \in X$ ) we obtain

$$
\mathfrak{u}(f) \leq \varphi_{L}\left(g_{1}\right)-\varphi\left(g_{2} \mathbb{1}_{A_{k}}\right) \quad(k \in \mathbb{N}),
$$

from which we conclude $\mathfrak{u}(f) \leq \varphi_{L}(g)$.

\section{Theorem 8.2}

(a) If $g \in S_{L V}$ and $f \leq g$, then $\mathfrak{u}(f) \leq \varphi_{L V}(g)$.

(b) If $S_{V}$ is stable, $g \in S_{V L V}$ and $f \leq g$, then $\mathfrak{u}(f) \leq \varphi_{V L V}(g)$.

Proof Follow the lines of the proof of the lemma with $S_{V}, S_{L}$ or $S_{V L}$ instead of $S$.

8.3 [Comments on Theorem 8.2]

(1) The theorem supersedes the lemma because $S_{V}+S_{L} \subset S_{L V}$.

\footnotetext{
${ }^{8}$ In this section we close an eye for the difference between a function and its equivalence class. There will be no danger of confusion.
} 
(2) As a consequence, $\mathfrak{u}=\varphi_{L V}$ on $\mathfrak{U} \cap S_{L V}$, and $\mathfrak{u}=\varphi_{V L V}$ on $\mathfrak{U} \cap S_{V L V}$ if $S_{V}$ is stable.

(3) Recall that stability of $S_{V}$ is necessary for the existence of $S_{V L V}$.

Theorem 8.4 Let E be a uniformly complete Riesz space and $\mathbb{B}$ be the Banach cover of principal ideals (see Example 5.5). $\mathfrak{U}$ is a linear subspace of $S_{L V}$ and $\mathfrak{u}=\varphi_{L V}$ on $\mathfrak{U}$.

Proof Let $u \in E^{+}$and let $f: X \rightarrow E_{u}$ be Bochner integrable. We prove $f \in S_{L V}$.

For simplicity of notation, put $D=E_{u}$. Let $S^{D}$ be the space of simple functions $X \rightarrow D$. By [16, Corollary 9.8] we have $f \in\left(S^{D}\right)_{L V}$ and $\mathfrak{u}(f)=\varphi_{L V}(f)$. Since $D$ is a Riesz ideal in $E$, the identity map $D \rightarrow E$ is order continuous. Then [16, Theorem 8.14] implies $f \in S_{L V}$.

Contrary to Theorem 8.4, in [16, Example 9.9(II)] $\mathfrak{U}$ is not a linear subspace of $S_{L V}$. Then next example shows, in the context of Theorem 8.4, that the inclusion may be strict.

Example 8.5 (U $\left.\subsetneq S_{L V}\right)$ For $X=\mathbb{N}, \mathcal{A}=\mathcal{P}(\mathbb{N})$ and $\mu$ the counting measure and $E=c$. As is mentioned in [16, Examples 9.9(I)], the function $n \mapsto \mathbb{1}_{\{n\}}$ is an element of $S_{L V}$ but not Bochner integrable. With $\mathbb{B}$ the Banach cover of principal ideals, the function $n \mapsto \mathbb{1}_{\{n\}}$ is not $\mathbb{B}$-integrable (see 5.10(4)).

\section{An example: convolution}

To illustrate the $\mathbb{B}$-integral as an extension of the Bochner integral we consider the following situation. (This introduction requires some knowledge of harmonic analysis on locally compact groups, the balance of this section does not.)

Let $G$ be a locally compact group. For $f: G \rightarrow \mathbb{R}$ and $x \in G$ we let $L_{x} f: G \rightarrow \mathbb{R}$ be the function $y \mapsto f\left(x^{-1} y\right)$.

For a finite measure $\mu$ on $G$ and $f$ in $L^{1}(G)$ one defines their convolution product to be the element $\mu * f$ of $L^{1}(G)$ given for almost every $y \in G$ by

$$
(\mu * f)(y)=\int f\left(x^{-1} y\right) \mathrm{d} \mu(x)=\int\left(L_{x} f\right)(y) \mathrm{d} \mu(x) .
$$

The map $x \mapsto L_{x} f$ of $G$ into $L^{1}(G)$ is continuous and bounded, hence Bochner integrable with respect to $\mu$. It is not very difficult to prove that

$$
\mu * f=\int L_{x} f \mathrm{~d} \mu(x)
$$

Similar statements are true for other spaces of functions instead of $L^{1}(G)$, such as $L^{p}(G)$, with $1<p<\infty$, and $C_{0}(G)$, the space of continuous functions that vanish at infinity.

But consider the space $C(G)$ of all continuous functions on $G$. The integrals $\int f\left(x^{-1} y\right) \mathrm{d} \mu(x)$ will not exist for all $f \in C(G), y \in G$ and all finite measures 
$\mu$, but they do if $\mu$ has compact support. Thus, one can reasonably define $\mu * f$ for $f \in C(G)$ and compactly supported $\mu$. However, there is no natural norm on $C(G)$ (except, of course, if $G$ is compact), so we cannot speak of $\int L_{x} f \mathrm{~d} \mu(x)$ as a Bochner integral. We will see that, at least for $\sigma$-compact $G$, it is a $\mathbb{B}$-integral where $\mathbb{B}$ is the Banach cover of $C(G)$ that consists of the principal (Riesz) ideals.

Theorem 9.1 Let $G$ be a $\sigma$-compact locally compact group. For every $f \in C(G)$ there exists a $w \in C(G)^{+}$such that

Every $L_{x} f \quad(x \in G)$ lies in the principal ideal $C(G)_{w}$; $x \mapsto L_{x} f$ is continuous relative to $\|\cdot\|_{w}$.

Proof Choose compact $K_{1} \subset K_{2} \subset \cdots$ such that $K_{1}$ is a neighbourhood of $e$; $K_{n}=K_{n}^{-1} ; K_{n} K_{n} \subset K_{n+1}, \bigcup_{n \in \mathbb{N}} K_{n}=G$.

For $x \in G$, define $[x]$ to be the smallest $n$ with $x \in K_{n}$. Then $[x]=\left[x^{-1}\right]$, $[x y] \leq 1+[x] \vee[y]$ for all $x, y \in G$ by definition.

Let $f \in C(G)$. Define $u, v: G \rightarrow[1, \infty)$ as follows:

$$
u(x):=1+\sup \left|f\left(K_{n+1}\right)\right|, \quad v(x)=[x] u(x) \quad \text { if }[x]=n .
$$

(1) If $x, y \in G,[x] \leq[y]=n$, then $x, y \in K_{n}$, so

$$
|f(x y)| \leq \sup \left|f\left(K_{n+1}\right)\right| \leq u(y) .
$$

(2) Hence, for all $x, y \in G$ : $\left|f\left(x^{-1} y\right)\right| \leq u(x) \vee u(y) \leq u(x) v(y)$, i.e., $\left|L_{x} f\right| \leq$ $u(x) v$.

Let $a \in G, \varepsilon>0$. In (5), using (3) and (4), we show the existence of a neighbourhood $U$ of $e$ with

$$
x \in a U \Longrightarrow\left|L_{x} f-L_{a} f\right| \leq \varepsilon v .
$$

Choose a $p$ with $a \in K_{p}$.

(3) $K_{1}$ contains an open set $V$ containing $e$. We make a $q \in \mathbb{N}$ with

$$
x \in a V \Longrightarrow\left|L_{x} f-L_{a} f\right| \leq \varepsilon v \text { on } G \backslash K_{q} .
$$

Let $x \in a V$. Then $x, a \in K_{p+1}$, so $[x],[a] \leq p+1$. By (1):

$$
\left|\left(L_{x} f-L_{a} f\right)(y)\right| \leq\left|f\left(x^{-1} y\right)\right|+\left|f\left(a^{-1} y\right)\right| \leq 2 u(y) \text { if }[y] \geq p+1 .
$$

Moreover, $\varepsilon v(y)=\varepsilon[y] u(y) \geq 2 u(y)$ if $[y] \geq \frac{2}{\varepsilon}$. Take $q \in \mathbb{N}$ with $q \geq p+1$ and $q \geq \frac{2}{\varepsilon}$. For $y \in G \backslash K_{q}$ we have $[y]>q$, so $\left|\left(L_{x} f-L_{a} f\right)(y)\right| \leq \varepsilon v(y)$.

(4) We show there exists an open set $W$ containing $e$ with

$$
x \in a W \Longrightarrow\left|L_{x} f-L_{a} f\right| \leq \varepsilon v \text { on } K_{q}
$$


The function $(x, y) \mapsto\left|\left(L_{x} f-L_{a} f\right)(y)\right|$ on $G \times G$ is continuous and $K_{q}$ is compact. Hence by [16, Theorem 8.15] the function $G \mapsto[0, \infty)$

$$
x \mapsto \sup _{y \in K_{q}}\left|\left(L_{x} f-L_{a} f\right)(y)\right|
$$

is continuous. Its value at $a$ is 0 , so there exists an open set $W$ containing $e$ with

$$
\sup _{y \in K_{q}}\left|\left(L_{x} f-L_{a} f\right)(y)\right| \leq \varepsilon \quad(x \in a W) .
$$

As $v(y) \geq 1$ for all $y$ we obtain (43).

(5) With $U=V \cap W$ we have

$$
x \in a U \Longrightarrow\left|L_{x} f-L_{a} f\right| \leq \varepsilon v \text { on } G .
$$

Therefore, to prove the theorem it is sufficient to show there exists a continuous function $w \geq v$ :

(6) Set $\alpha_{n}=n\left(1+\sup \left|f\left(K_{n+1}\right)\right|\right)$ for all $n$; then

$$
[x]=n \Longrightarrow v(x)=\alpha_{n} \text {. }
$$

Put $K_{0}=\emptyset$. For all $n \in \mathbb{N}$ we have $V K_{n-1} \subset K_{1} K_{n-1} \subset K_{n}$, so $K_{n-1}$ is a subset of $K_{n}^{\circ}$, the interior of $K_{n}$. By Urysohn [8, Theorem VII.4.1 and Theorem XI.1.2] for all $n$ there is a continuous $g_{n}: G \rightarrow[0,1]$ with

$$
g_{n}=0 \text { on } K_{n-1}, g_{n}=1 \text { on } G \backslash K_{n}^{\circ} \supset G \backslash K_{n} .
$$

Let $b \in G$. There is a $l$ with $b \in K_{l} . b V$ is an open set containing $b$. As $b V \subset K_{l+1}$ and $g_{n}=0$ on $K_{n-1}$ we have: $g_{n}=0$ on $b V$ as soon as $n \geq l+2$.

Hence, $w:=\sum_{n \in \mathbb{N}} \alpha_{n+1} g_{n}$ is a continuous function $G \rightarrow[0, \infty)$. For every $x \in G$ there is an $n$ with $[x]=n$; then $x \notin K_{n-1}, g_{n-1}(x)=1$ and $w(x) \geq \alpha_{n}=$ $v(x)$.

Theorem 9.2 Let $G$ be a $\sigma$-compact locally compact group. Let $\mu$ be a finite measure on the Borel $\sigma$-algebra of $G$ with a compact support. Let $\mathbb{B}$ be the Banach cover of $C(G)$ consisting of the principal ideals as in Example 5.5. Then for every $f \in C(G)$ the function $x \mapsto L_{x} f$ is $\mathbb{B}$-integrable and its integral is the "convolution product" $\mu * f:$

$$
(\mu * f)(y)=\int f\left(x^{-1} y\right) \mathrm{d} \mu(x) \quad(y \in G) .
$$

Proof By Theorem 9.1 there exists a $w \in C(G)^{+}$such that (37) holds. This implies that the map $x \mapsto L_{x} f$ is Borel measurable and $\left\{L_{x} f: x \in G\right\}$ is separable in $C(G)_{w}$. As $x \mapsto\left\|L_{x} f\right\|_{w}$ is continuous and thus bounded on the support of $\mu$, the map $x \mapsto L_{x} f$ is $\mathbb{B}$-integrable (see 4.4(a)). That the integral is equal to $\mu * f$ follows by $4.4(\mathrm{c})$. 
Remark 9.3 Theorem 9.2 compares to [16, Example 8.17] in the sense that in both situations the convolution is equal to an integral of the translation. Though the situation is slightly different in the sense that in Theorem 9.2 we consider $\sigma$-compact locally compact groups, while in [16, Example 8.17] we considered metric locally compact groups (the fact that in [16, Example 8.17] $L_{x} f(t)=f\left(t x^{-1}\right)$ is reminiscent for its statement).

Acknowledgements W.B. van Zuijlen is supported by the ERC Advanced Grant VARIS-267356 of Frank den Hollander.

Open Access This article is distributed under the terms of the Creative Commons Attribution 4.0 International License (http://creativecommons.org/licenses/by/4.0/), which permits unrestricted use, distribution, and reproduction in any medium, provided you give appropriate credit to the original author(s) and the source, provide a link to the Creative Commons license, and indicate if changes were made.

\section{A Appendix: Absolutely dominating norms}

\section{In this section, $C>0$ and $D$ is an ordered Banach space with closed generating cone and with a $\boldsymbol{C}$-absolutely dominating norm $\|\cdot\|$.}

As is mentioned in 3.5, we show that $C \geq 1$ (A.1) and that for every $\varepsilon>0$ there exists an equivalent $(1+\varepsilon)$-absolutely norm (Theorem A.3). Furthermore, we discuss (in A.4-A.10) whenever there exists an equivalent norm $\|\cdot\|_{1}$ for which

$$
\|x\|_{1}=\inf \left\{\|a\|_{1}: a \in D^{+},-a \leq x \leq a\right\} \quad(x \in D) .
$$

This is done by means of the norm $\mathcal{N}$ introduced in A.2. Example A.11 illustrates that the existence of such equivalent norm may fail.

A.1 $\left[C\right.$ has to be $\geq 1$ ] Suppose that $C<1$. Choose $C^{\prime}>0$ such that $C<C^{\prime}<1$. For all $a \in D^{+}$with $a \neq 0$ there exists a $b \in D^{+}$with $a \leq b$ and $\|b\| \leq C^{\prime}\|a\|$. Let $a \in D^{+}$with $\|a\|=1$. Iteratively one obtains a sequence $a \leq a_{1} \leq a_{2} \leq \cdots$ with $\left\|a_{1}\right\| \leq C^{\prime}$ and $\left\|a_{n+1}\right\| \leq C^{\prime}\left\|a_{n}\right\|$ for all $n$. Then $\sum_{n \in \mathbb{N}}\left\|a_{n}\right\|<\infty$ and thus $a_{n} \stackrel{u}{\rightarrow} 0$ by Theorem 3.9, which contradicts $0<a \leq a_{n}$.

A.2 Define $\mathcal{N}: D \rightarrow[0, \infty)$ by

$$
\mathcal{N}(x)=\inf \left\{\|a\|: a \in D^{+},-a \leq x \leq a\right\} .
$$

$\mathcal{N}$ is a seminorm, and actually a norm because (see Theorem 3.9)

$$
\mathcal{N}(x)=0 \Longleftrightarrow \text { there is an } a^{*} \in D^{+} \text {with }-\frac{1}{n} a^{*} \leq x \leq \frac{1}{n} a^{*} \quad(n \in \mathbb{N}) .
$$

Because $\|\cdot\|$ is $C$-absolutely dominating one has $\mathcal{N} \leq C\|\cdot\|$.

Theorem A.3 For all $\varepsilon>0$ there exists an equivalent norm $\rho$ on $D$, for which

$$
(1+\varepsilon)^{2} \rho(x) \geq \inf \left\{\rho(a): a \in D^{+},-a \leq x \leq a\right\} \quad(x \in D) .
$$


Proof Define $\rho:=\varepsilon \mathcal{N}+\|\cdot\| . \rho$ is a norm which is equivalent to $\|\cdot\|$, since $\mathcal{N} \leq C\|\cdot\|$. Let $x \in D, x \neq 0$. Choose $a \in D^{+}$with $-a \leq x \leq a$ such that $\|a\| \leq(1+\varepsilon) \mathcal{N}(x)$.

Note that by definition of $\mathcal{N}$ we have $\mathcal{N}(a) \leq\|a\|$. Whence

$$
\frac{\rho(a)}{\rho(x)}=\frac{\mathcal{N}(a)+\varepsilon\|a\|}{\mathcal{N}(x)+\varepsilon\|x\|} \leq \frac{\mathcal{N}(a)+\varepsilon\|a\|}{\mathcal{N}(x)} \leq \frac{\|a\|+\varepsilon\|a\|}{\mathcal{N}(x)} \leq(1+\varepsilon) \frac{\|a\|}{\mathcal{N}(x)} \leq(1+\varepsilon)^{2} .
$$

A.4 Suppose $\|\cdot\|_{1}$ is a norm equivalent to $\|\cdot\|$ for which there exists a $C>0$ such that

$$
C\|x\|_{1}=\inf \left\{\|a\|_{1}: a \in D^{+},-a \leq x \leq a\right\} \quad(x \in D) .
$$

Then it is straightforward to show that $\mathcal{N}$ is equivalent to $\|\cdot\|$.

A.5 $\mathcal{N}$ is equivalent to $\|\cdot\|$ if and only if there exists a $c>0$ such that $\mathcal{N} \geq c\|\cdot\|$. The latter is true if and only if

$$
-\frac{1}{n} a^{*} \leq x_{n} \leq \frac{1}{n} a^{*} \quad(n \in \mathbb{N}) \Longrightarrow \lim _{n \rightarrow \infty}\left\|x_{n}\right\|=0 .
$$

\section{Theorem A.6}

$$
\mathcal{N}(x)=\inf \left\{\mathcal{N}(a): a \in D^{+},-a \leq x \leq a\right\}
$$

Proof $(\leq)$ Take $a \in D^{+},-a \leq x \leq a$; we prove $\mathcal{N}(x) \leq \mathcal{N}(a)$. For all $b \geq a,-b \leq$ $x \leq b$, whence $\mathcal{N}(x) \leq\|b\|$. Thus $\mathcal{N}(x) \leq \inf \{\|b\|: a \leq b\}=\mathcal{N}(a)$, the latter by definition of $\mathcal{N}(a)$.

$(\geq)$ Take $\varepsilon>0$. Choose $a \in D^{+},-a \leq x \leq a,\|a\| \leq \mathcal{N}(x)+\varepsilon$. Then $\mathcal{N}(a) \leq$ $\|a\| \leq \mathcal{N}(x)+\varepsilon$.

Theorem A.7 [1, Theorem 2.38] For an ordered normed vector space E the following are equivalent.

(a) The cone $E^{+}$is normal.

(b) The normed space $E$ admits an equivalent monotone norm.

(c) There is a $c>0$ such that $0 \leq x \leq y$ implies $\|x\| \leq c\|y\|$.

A.8 Suppose $D$ is an ordered Banach space with closed generating cone and suppose there exists a $c>0$ such that

$$
\|x\| \leq c \inf \left\{\|a\|: a \in D^{+},-a \leq x \leq a\right\}=c \mathcal{N}(x) .
$$

Then for $x \in D^{+}, a \in D^{+}$with $x \leq a$ one has $\|x\| \leq c\|a\|$.

A.9 Suppose $D$ is an ordered Banach space with closed generating cone and $\|\cdot\|$ is monotone. Let $x \in D$ and $a \in D^{+}$be such that

$$
-a \leq x \leq a .
$$


Then $0 \leq x+a \leq 2 a$ and whence $\|x\| \leq\|x+a\|+\|a\| \leq 3\|a\|$. Thus

$$
\|x\| \leq 3 \inf \left\{\|a\|: a \in D^{+},-a \leq x \leq a\right\}=3 \mathcal{N}(x) .
$$

We conclude:

Theorem A.10 Let D be an ordered Banach space with closed generating cone. The following are equivalent

(a) There exist a norm $\|\cdot\|_{1}$ that is equivalent to $\|\cdot\|$ for which

$$
\|x\|_{1}=\inf \left\{\|a\|_{1}: a \in D^{+},-a \leq x \leq a\right\} \quad(x \in D) .
$$

(b) There exists a $c>0$ such that $\mathcal{N} \geq c\|\cdot\|$.

(c) There exists a monotone norm that is equivalent to $\|\cdot\|$.

(d) $E^{+}$is normal.

In the following we give an example of an ordered Banach space with closed generating cone $D$ for which none of (a)-(d) of Theorem A.10 holds.

Example A.11 Let $D$ be $\ell^{1}$ with its natural norm. Define $T: \ell^{1} \rightarrow \mathbb{R}^{\mathbb{N}}$ by

$$
T x=\left(x_{1}, x_{1}+x_{2}, x_{1}+x_{2}+x_{3}, \ldots\right) .
$$

As $T$ is linear, $D$ is an ordered vector space under the relation $\preceq$

$$
x \preceq y \Longleftrightarrow T x \leq T y .
$$

The positive cone of $\ell^{1}$ is included in $D^{+}$, whence $D$ is directed. Moreover, $D^{+}$is closed and so $\|\cdot\|$ is absolutely dominating. With $x_{n}=(1,-1,1,-1, \ldots, \pm 1,0,0 \ldots)$ and $a=(1,0,0, \ldots)$ we have $-a \leq x_{n} \leq a$ and $\left\|x_{n}\right\|=n,\|a\|=1$.

\section{B Appendix: Banach cover of Köthe spaces}

In this section $(Y, \mathcal{B}, v)$ is a complete $\sigma$-finite measure space and $M$ is the space of classes of measurable functions $Y \rightarrow \mathbb{R}$ as in Example 5.6.

Lemma B.1 $\left\{L_{\rho_{w}}: w \in M^{+}, w>0\right\}$ a.e. is a Banach cover of $M$.

Proof $L_{\rho_{w}}$ is complete since $f \mapsto f w$ is an isometric bijection $M_{w} \rightarrow L^{1}(v)$.

Let $f \in M$. We show there exists a $w \in M^{+}, w>0$ a.e., with $f \in L_{\rho_{w}}$. By the $\sigma$-finiteness of $v$ there is a $u \in L^{1}(v), u>0$ a.e. Put $w=(|f|+1)^{-1} u$. Then $w \in M^{+}, w>0$ a.e., and $f \in L_{\rho_{w}}$ because $\int|f| w \mathrm{~d} v \leq \int u \mathrm{~d} v<\infty$.

If $w, v \in M^{+}, w>0, v>0$ a.e., then $w \wedge v>0$ a.e., $L_{\rho_{w \wedge v}}$ is a subset of $L_{\rho_{w}}$ and $L_{\rho_{v}}$ and $\rho_{w \wedge v} \leq \rho_{w}$ on $L_{\rho_{w}}$ and $\rho_{w \wedge v} \leq \rho_{v}$ on $L_{\rho_{v}}$.

Theorem B.2 The complete Köthe spaces form a Banach cover of $M$. 
Proof Let $\rho$ be a function norm and $L_{\rho}$ be complete. If $f_{n} \in M^{+}$for $n \in \mathbb{N}$ and $\sum_{n \in \mathbb{N}} \rho\left(f_{n}\right)<\infty$, then $\sum_{n \in \mathbb{N}}\left|f_{n}\right| \in L_{\rho}$, so $\sum_{n \in \mathbb{N}}\left|f_{n}\right|<\infty$ a.e. and $f_{n} \rightarrow 0$ a.e. We will use this fact below.

By Lemma B.1 it suffices to prove the following. Let $\rho_{1}, \rho_{2}$ be function norms, $L_{\rho_{1}}$ and $L_{\rho_{2}}$ complete. We make a function norm $\rho$ such that $L_{\rho}$ is complete and $\rho \leq \rho_{1}$, $\rho \leq \rho_{2}$. (Then $L_{\rho_{1}}, L_{\rho_{2}} \subset L_{\rho}$ and we are done.)

Define $\rho: M \rightarrow[0, \infty]$ by

$$
\rho(f)=\inf \left\{\rho_{1}(g)+\rho_{2}(h): g, h \in M^{+}, g+h \geq|f|\right\} .
$$

If $\rho(f)=0$, choose $g_{n}, h_{n}$ with $g_{n}+h_{n} \geq|f|, \rho_{1}\left(g_{n}\right)+\rho_{2}\left(h_{n}\right) \leq 2^{-n}$. Then (by the above), $g_{n} \rightarrow 0$ a.e., $h_{n} \rightarrow 0$ a.e. Hence, $f=0$ a.e. It follows easily that $\rho$ is a function norm. Obviously, $\rho \leq \rho_{1}, \rho \leq \rho_{2}$. For the completeness of $L_{\rho}$ : Let $u_{1}, u_{2}, \ldots \in L_{\rho}^{+}, \sum_{n \in \mathbb{N}} \rho\left(u_{n}\right)<\infty$. Choose $g_{n}, h_{n} \in M^{+}, g_{n}+h_{n} \geq u_{n}$, $\rho_{1}\left(g_{n}\right)+\rho_{2}\left(h_{n}\right)<\rho\left(u_{n}\right)+2^{-n}$. Then $\sum_{n \in \mathbb{N}} \rho_{1}\left(g_{n}\right)<\infty$, so $\sum_{n \in \mathbb{N}} g_{n} \in L_{\rho_{1}}$, $\rho_{1}\left(\sum_{n \in \mathbb{N}} g_{n}\right)<\infty$. Similarly $\rho_{2}\left(\sum_{n \in \mathbb{N}} h_{n}\right)<\infty$. Then $\sum_{n \in \mathbb{N}} u_{n} \in L_{\rho}$.

\section{References}

1. Aliprantis, C.D., Tourky R.: Cones and Duality, volume 84 of Graduate Studies in Mathematics. American Mathematical Society (2007)

2. Andô, T.: On fundamental properties of a Banach space with a cone. Pac. J. Math. 12, 1163-1169 (1962)

3. Batty, C.J.K., Robinson, D.W.: Positive one-parameter semigroups on ordered Banach spaces. Acta Applicandae Mathematicae 2(3), 221-296 (1984)

4. Birkhoff, G.: Lattice Theory, volume XXV of Colloquium publications. American Mathematical Society, third edn (1967)

5. Б. З. Вулих. Введение в теорию конусов в нормированных пространствах. Изд-во Калинин. ун-та, pages 1-84, 1977. B.Z. Wulich. Einführung in die Theorie der Kegel in normierten Räumen. (Russisch). Izd. Staatl. Universität Kalinin. 1977 (handschriftl. deutsche Übersetzung: M.R.Weber)

6. Б. 3. Вулих. Специальные вопросы геометрии конусов в нормированных пространствах. Изд-во Калинин. ун-та , pages 1-84, 1978. B.Z. Wulich. Spezielle Probleme der Geometrie von Kegeln in normierten Räumen. (Russisch). Izd. Staatl. Universität Kalinin. 1978 (handschriftl. deutsche Übersetzung: M.R.Weber)

7. Conway, J.B.: A Course in Functional Analysis, second edn. Springer (2007)

8. Dugundji, J.: Topology. Allyn and Bacon series in Advanced Mathematics. Allyn and Bacon Inc (1966)

9. Hille, E., Phillip, R.S.: Functional Analysis and Semi-Groups, volume 31 of Colloquium publications. American Mathematical Society (1957)

10. Hogbe-Nlend, H.: Bornologies and Functional Analysis: Introductory Course on the Theory of Duality Topology-bornology and Its Use in Functional Analysis, volume 62 of Notas de matemática. NorthHolland Publishing Company (1977)

11. de Jonge, E., van Rooij A.C.M.: Introduction to Riesz spaces. Mathematisch Centrum (1977)

12. Luxemburg, W.A.J., Zaanen, A.C.: Riesz spaces, vol. I. North-Holland Mathematical Library. NorthHolland Publishing Co., Amsterdam-London; American Elsevier Publishing Co., New York (1971)

13. Messerschmidt, M.: Normality of spaces of operators and quasi-lattices. Positivity 19(4), 695-724 (2015)

14. Namioka, I.: Partially ordered linear topological spaces. vol. 24, pp. 49. American Mathematical Society, USA (1957)

15. Peressini, A.L.: Ordered Topological Vector Spaces. Harper \& Row Publishers, New York (1967)

16. van Rooij, A., van Zuijlen, W.: Integrals for functions with values in partially ordered vector spaces. Positivity 20(4), 877-916 (2016) 
17. Ryan, R.A.: Introduction to tensor products of Banach spaces. Springer Monographs in Mathematics. Springer, London Ltd, London (2002)

18. Thomas, G.E.F.: Integration of functions with values in locally convex suslin spaces. Trans. Am. Math. Soc. 212, 61-81 (1975)

19. Zaanen, A.C.: Riesz Spaces II. North-Holland Publishing Company (1983) 